\title{
Vancomycin Spacer-induced Hemolysis
}

\author{
Anuraj Sudhakaran ${ }^{1}$, Aparna Baburaj ${ }^{2}$, Kinjal Banerjee ${ }^{1}$, Rajesh Essrani ${ }^{1}$ \\ 1. Internal Medicine, Geisinger Medical Center, Danville, USA 2. Internal Medicine, Geisinger Medicine Center, \\ Danville, USA
}

Corresponding author: Anuraj Sudhakaran,dranurajms@yahoo.com

\begin{abstract}
Intravenous vancomycin-induced hemolysis has been documented in the literature. This has been presumed to be due to the development of antibodies against additives that are added along with antibiotics. Herein, we present the case of a 72-year-old male who had hemolysis after the placement of vancomycin spacer, which improved after the spacer was removed.
\end{abstract}

Categories: Internal Medicine, Infectious Disease, Nephrology

Keywords: vancomycin induced hemolysis, drug induced hemolysis, spacer induced hemolysis, auto immune hemolytic anemia, drug induced hemolytic anemia, hemolysis after orthopedic procedure

\section{Introduction}

Drug-induced immune hemolytic anemia (DIIHA) is often a rare and sometimes fatal side effect of some of the medications. These medications include but not limited cephalosporins, penicillin, nonsteroidal antiinflammatory drugs [1]. DIIHA associated with vancomycin is rare, and there are only very few reported cases of autoimmune hemolytic anemia caused by anti-vancomycin antibodies [2]. Herein, we present the case of autoimmune hemolytic anemia caused due to vancomycin spacer placed during an orthopedic intervention. DIIHA improved after spacer was removed.

\section{Case Presentation}

A 72-year-old male presented with a past medical history of type 2 diabetes mellitus, chronic kidney disease stage 3, and atrial fibrillation on warfarin. He underwent a total hip arthroplasty at an outside hospital, which was complicated by a post-operative infection. Per records, he was taken back to the operating room approximately one week after the index surgery. The joint was drained, and he was started on ceftriaxone for two months via a peripherally inserted central catheter (PICC) line, followed by six months of oral cefdinir. Approximately three weeks after the completion of oral antibiotics, he developed pain and swelling in the joint. A joint aspirate demonstrated cloudy brown fluid with 28,700 white blood corpuscles (WBC), strongly suspicious of a septic joint. A two-stage procedure was planned, and he was taken back to surgery, where the prosthesis was removed and replaced with a vancomycin spacer (containing vancomycin of $6 \mathrm{~g}$ ). The

Received 07/08/2019

Review began 07/15/2019 Review ended 09/20/2019 Published 10/06/2019

\section{() Copyright 2019}

Sudhakaran et al. This is an open access article distributed under the terms of the Creative Commons Attribution License CC-BY 3.0., which permits unrestricted use, distribution, and reproduction in any medium, provided the original author and source are credited. synovial fluid culture was positive for Enterobacter and Enterococcus. He was started on a planned six-week course of IV cefepime and vancomycin via PICC line. Approximately two weeks into his course of antibiotics, he was readmitted with drop-in hemoglobin from 8.5 to 5.7 and an acute kidney injury (creatinine $3.7 \mathrm{mg} / \mathrm{dl}$ from baseline of $1.6 \mathrm{mg} / \mathrm{dl}$ ). He was found to have a high vancomycin trough level (61.2 micrograms $/ \mathrm{ml}$ ), and IV vancomycin was stopped and replaced with IV daptomycin. His renal function continued to worsen, and hemodialysis was necessary. After initiation of HD, there were biochemical features of ongoing hemolysis and thrombocytopenia, raising concerns for thrombotic thrombocytopenic purpura (TTP) and atypical hemolytic uremic syndrome (aHUS). Renal biopsy, ADAMTS13, and aHUS genetic panels were ordered and showed negative results. He was started on plasmapheresis empirically but was discontinued after two days when ADAMTS13 resulted as normal. Renal biopsy showed acute tubular injury with hemoglobin-positive casts in the setting of chronic changes. He was diagnosed with vancomycininduced immune hemolytic anemia. He underwent surgical removal of the vancomycin spacer. After the removal of the spacer, his hemoglobin stabilized, and hemolytic parameters significantly improved. Although his renal function has not improved three months post-discharge, his hemoglobin, haptoglobin, and lactate dehydrogenase levels remained within normal limits.

\section{Discussion}

Drug-induced immune thrombocytopenia anemia rarely occurs one in million. There is a sudden decrease in hemoglobin levels after the patients are started on the presumed drug. The most common drugs associated with immune hemolytic anemia include cefotetan, ceftriaxone, and piperacillin [3]. Two postulates explain the interaction of these drugs leading to immune-mediated hemolysis. These drugs may alter the antigens on the red cell, resulting in the production of antibodies that cross-react with unaltered antigen, or the drugs may associate with structures on the red cell and be part of the antigen resulting in haptenic reaction [4]. Higher doses of penicillin cause hemolysis due to the formation of immune complexes [5]. Vancomycininduced hemolytic anemia has been reported recently. Vancomycin antibodies were detected in these cases. Very few reported cases of DIIHA associated with anti-vancomycin are currently available. In this case, 
hemolysis continued despite discontinuing IV vancomycin. Only after removing his vancomycin spacer did his hemolytic parameters improve.

Polymethylmethacrylate cement preloaded with antibiotics are used in some cases for prophylaxis and also for the surgical revision of prosthetic infections. These spacers help deliver a high concentration of antibiotics around the joint, which would have been difficult to achieve without significant systemic side effects [6]. Antibiotic concentration in serum was very low after the implantation of the spacer. In the study by Gniadek et al., vancomycin antibodies developed after starting patients on IV vancomycin led to hemolysis. As red blood cells (RBC) pretreated with vancomycin did not react with antibodies, Gniadek hypothesized that vancomycin does not covalently bind to the RBC surface leading to hemolysis. We believe that our patient developed anti-vancomycin antibodies during his initial intravenous therapy resulting in hemolysis. His hemolysis continued even after IV vancomycin was discontinued. The presence of vancomycin-impregnated spacers may have been the source of continued antibody-mediated hemolysis. His hemolytic parameters continued to improve and returned to normal after vancomycin spacer was removed. The cost for vancomycin antibody testing was prohibitive in our case and could not be done. The cessation of hemolysis and the lack of an alternative cause for hemolysis during the time period makes vancomycinimpregnated spacer to be responsible for his continued hemolysis.

\section{Conclusions}

Vancomycin-induced immune hemolytic anemia is a rare cause of hemolytic anemia. Patients with vancomycin-impregnated spacers can have continued hemolysis despite stopping IV vancomycin. There should be a high index of suspicion for continued antibody production in the presence of vancomycin spacers and should be promptly removed. Vancomycin antibody testing should be done if possible before removal.

\section{Additional Information \\ Disclosures}

Human subjects: Consent was obtained by all participants in this study. Conflicts of interest: In compliance with the ICMJE uniform disclosure form, all authors declare the following: Payment/services info: All authors have declared that no financial support was received from any organization for the submitted work. Financial relationships: All authors have declared that they have no financial relationships at present or within the previous three years with any organizations that might have an interest in the submitted work. Other relationships: All authors have declared that there are no other relationships or activities that could appear to have influenced the submitted work.

\section{References}

1. Johnson ST, Fueger JT, Gottschall JL: One center's experience: the serology and drugs associated with druginduced immune hemolytic anemia--a new paradigm. Transfusion. 2007, 47:697-702. 10.1111/j.15372995.2007.01173.x

2. Gniadek TJ, Arndt PA, Leger RM: Drug-induced immune hemolytic anemia associated with anti-vancomycin complicated by a paraben antibody. Transfusion. 2018, 58:181-188. 10.1111/trf.14362

3. Sarkar RS, Philip J, Mallhi RS: Drug-induced immune hemolytic anemia (Direct Antiglobulin Test positive) . Med J Armed Forces India. 2013, 69:190-192. 10.1016/j.mjafi.2012.04.017

4. Worlledge SM, Carstairs KC, Dacie JV: Autoimmune haemolytic anaemia associated with alpha-methyldopa therapy. Lancet. 1966, 2:135-139. 10.1016/s0140-6736(66)92423-8

5. Ries CA, Rosenbaum TJ, Garratty G: Penicillin-induced immune hemolytic anemia. Occurrence of massive intravascular hemolysis. JAMA. 1975, 233:432-435. 10.1001/jama.1975.03260050038017

6. Regis D, Sandri A, Samaila E: Release of gentamicin and vancomycin from preformed spacers in infected total hip arthroplasties: measurement of concentrations and inhibitory activity in patients' drainage fluids and serum. Scientific World Journal. 2013, 2013:752184. 10.1155/2013/752184 